\title{
Management of complications after reverse shoulder arthroplasty
}

\author{
Hanbing S. Zhou • Justin S. Chung • Paul H. Yi • \\ Xinning Li • Mark D. Price
}

Published online: 10 January 2015

(C) Springer Science+Business Media New York 2015

\begin{abstract}
Reverse total shoulder arthroplasty (RTSA) has become the treatment of choice for patients with rotator cuff arthropathy. Complication rate after RTSA has been reported to be three to five times that of conventional total shoulder arthroplasty. Intraoperative and postoperative complications include neurological injury, infection, dislocation or instability, acromial or scapular spine fracture, hematoma, and scapular notching. Knowledge of optimal component placement along with preoperative planning and recognition of risk factors are essential in optimizing patient outcome. The purpose of this review article is to identify the most common and serious complications associated with the RTSA and discuss the current methods of management. Complications after RTSA pose a significant challenge for healthcare providers and economic burden to society. Therefore, it is essential to make the proper diagnosis and develop and implement early management plans to improve patient outcome and satisfaction.
\end{abstract}

This article is part of the Topical Collection on Shoulder Surgery: Complications

H. S. Zhou $\cdot$ M. D. Price $(\bowtie)$

Department of Orthopaedic Surgery, University of Massachusetts

Medical Center, Worcester, MA, USA

e-mail: Mark.Price@umassmemorial.org

H. S. Zhou

e-mail: hanbing.zhou@gmail.com

J. S. Chung $\cdot$ X. Li

Department of Orthopaedic Surgery, Boston University School of

Medicine, Boston, MA, USA

P. H. Yi

Department of Orthopaedic Surgery, University of California San Francisco, San Francisco, CA, USA
Keywords Reverse shoulder arthroplasty · Complication · Management

\section{Introduction (reverse total shoulder arthroplasty, complications)}

The development of the reverse total shoulder arthroplasty (RTSA) in the late 1980s provided orthopedic surgeons with an alternative to total shoulder arthroplasty (TSA) for the treatment of rotator cuff tears and arthropathy. Conventional TSA relies on the anatomical and functional integrity of the rotator cuff muscles. This procedure can often be ineffective in patients with massive rotator cuff tears and is associated with high complication rates [1]. In contrast, RTSA is able to restore functionality for patients with cuff tear arthropathy or in patients with failed conventional total shoulder replacement by virtue of the unique design [1]: the RTSA reverses the shoulder joint by fixing a metal ball to the glenoid and introducing a spherical socket into the proximal end of the humerus [2]. This new construct lowers the humerus and medializes the center of rotation to decrease torque at the glenoid component and recruit the deltoid muscle fibers rather than rotator cuff muscles to facilitate arm flexion and drive upper extremity function [3]. The ball and socket design with increased contact surface area provide increased stability or constraint to the shoulder joint.

Despite the benefits of the RTSA, patients who undergo this procedure are often subject to complications that are similar to conventional TSA as well as unique to RTSA because of the major anatomical, physiological, and biomechanical changes to the shoulder joint. The purpose of the present review is to describe the major potential complications following RTSA and to provide an overview of management of such complications. The complications to be covered 
include infection, nerve injury, intraoperative periprosthetic fracture, hematoma, dislocation, scapular notching, acromial fracture, and complications relating to the glenoid or humeral components.

\section{Complications}

\section{Nerve injury}

The most common neurological injuries as a result of RTSA procedure involve the brachial plexus or the axillary nerve [4]. This could be attributed to several intraoperative factors: traction, manipulation of the arm, aberrant retractor placement, or relative lengthening of the arm [2]. Furthermore, during the glenoid exposure, the humerus is posteriorly retracted, externally rotated, and abducted, resulting in excessive traction on the brachial plexus and axillary nerve in particular [2]. Electromyography (EMG) should be obtained after 6 weeks from the onset of symptoms to document the nerve injury (neuropraxia vs. transection) and should be repeated at 6 months to 1 year to follow improvement.

In addition, radial nerve palsy has been reported in the literature in RTSA for treatment of failed hemiarthroplasty for proximal humerus fractures. In this case, the patient had subsequent resolution of symptoms with conservative management [5]. Fortunately, these neurological injuries are relatively rare and majority of them are reversible within the first three postoperative months [6].

While intraoperative manipulation of the arm certainly can lead to nerve palsy, the actual design of the implant itself can lead to problems as well. Because insufficient deltoid tension can lead to prosthetic instability, reverse implants are designed to distalize the center of rotation and increase the humeral length in order to generate higher deltoid tension and stability, which can also put undue stress on the nerves of the upper extremity. Van Hoof et al. used a three-dimensional computer model of a RTSA construct and estimated that the insertion of prosthesis can lead to 15.3 and $19.3 \%$ increase in strain on the lateral and medial roots of the median nerve, respectively [7]. Similarly, Boileau et al. [8] reported 1 case of axillary nerve palsy in a study of 45 consecutive patients who had RTSA with the original Grammont (Delta III; Depuy) prosthesis. Muh et al. [9•] reported 1 case of radial and ulnar nerve palsy in a series of 67 RTSA procedure using Grammont (Tornier, France) prosthesis. The patient had incomplete resolution of symptoms at 30 months follow-up. Wingert et al. [10] reported 1 patient with persistent radial, ulnar, and musculocutaneous nerve injury after intraoperative axillary artery avulsion and placement of synthetic arterial graft. Fortunately, most of these neurological complaints are transient and should be treated with observation alone.
In a cadaver study, Ladermann et al. [11] demonstrated that after implantation of the reverse shoulder components, the distance of the axillary nerve was $>15 \mathrm{~mm}$ from the glenosphere; however, the main anterior branch of the axillary nerve was in close contact with the posterior metaphysis or humeral component of the reverse shoulder prosthesis $(5.2 \pm$ $2.1 \mathrm{~mm}$ ). They theorized that with distalization of the center of rotation, the axillary nerve is lowered and lateralized from the glenosphere component, which protects it from impingement. This altered position places the axillary nerve in close proximity to the posterior humeral component and may therefore increase the risk of injury. The authors recommended caution when reaming the humeral metaphysis and also avoid retroversion greater than 20 to $30^{\circ}$.

\section{Intraoperative fracture}

Intraoperative fractures can occur on either the humeral or the glenoid side. They are relatively uncommon but can be challenging to manage. Intraoperative humeral fractures mainly occurred during revision surgeries in the process of removing the primary stem or the associated cement mantle. Commonly used techniques such as avoiding excessive torque and reaming the canal by hand can minimize the chances of intraoperative humeral fracture. If the fracture occurred prior to humeral implantation, then it should be stabilized using cerclage wiring, allograft strut augmentation, or plate and screws in addition to a longer stem to bypass the fracture site $[2,12]$. If the fracture occurred after humeral stem implantation and the implant is stable, then the fracture should be fixed using a combination of cerclage wires or plates and screws [12].

Intraoperative glenoid fractures can be a result of the reaming process or the fixation technique. Care must be taken during the reaming process to ensure the reamer is started on full speed prior to contacting the glenoid and it does not ream beyond the subchondral bone so that adequate bone stock may be preserved [12]. If intraoperative glenoid fracture does occur, fragment-specific fixation or redirecting the baseplate and screws may create a strong fixation for a stable glenosphere component. In the case of catastrophic glenoid failure, a two-stage procedure with glenoid bone grafting (allograft or iliac crest) and reimplantation of the components after bone incorporation into the glenoid. Alternatively, complete resection of the glenopshere component and conversion of the reverse arthroplasty to a large head hemiarthroplasty or complete resection arthroplasty may be performed, which will result in suboptimal patient outcomes. Boileau et al. [8] reported 1 case of humeral fracture and 1 case of glenoid fracture in a series of 45 patients. In a review article published by Zumstein et al., the rate of intraoperative humeral fracture was $2.0 \%(16 / 782)$, and the intraoperative glenoid fracture was $0.9 \%(7 / 782)[13 \bullet \bullet$. 


\section{Infection}

Infection rate after RTSA has a reported incidence that ranges from 1 to $10 \%$ [2, 14-18]. Zumstein et al. reported the incidence of deep infection to be $3.8 \%$ (30/782), which was similar other arthroplasty procedures but higher than other shoulder surgeries [13••]. The incidence of infection was statistically higher in revision cases than primary cases (5.9 vs. $2.9 \%$ ). The most common offending organisms were low virulence in nature, including Priopionibacterium acnes and Staphylococcus epidermidis [19, 20]. Treatment for RTSA infection is similar to other joint arthroplasty procedures. Preoperative antibiotics should be administered within $1 \mathrm{~h}$ of incision and antibiotic-impregnated cement may substantially decrease the rate of infection especially in patients who are high risk [21]. In the case that postoperative infection does occur, antibiotic infusion should be started only after cultures are taken to allow speciation of the bacteria and antibiotics should tailored accordingly. Acute infection ( $<6$ weeks) should be treated with thorough irrigation, debridement, and polyethylene exchange [2]. In the case of chronic infection, a two-stage complete revision approach may be warranted. Stage 1 includes hardware removal, irrigation and debridement, and antibiotic spacer placement with at least 6 weeks of culture-specific antibiotics [2]. Stage 2 involves prosthesis reimplantation, after confirmation of elimination of the infection, often done by image-guided aspiration of the glenohumeral joint. Beekman et al. [22] reported successful one-stage revision to limit the amount of anesthesia and the morbidity of a second procedure to the patient. Zavala et al. [20] reported successful treatment of infected RTSA with a combination debridement, liner/glenosphere exchange, and IV antibiotics regardless of chronicity of the infection. Traditional staged resection arthroplasty can lead to significant bone and soft tissue loss and should be reserved for persistent infections or significant bone stock loss unsuitable for reimplantation [20, 22]. Utilization of an open biopsy prior to the reimplantation stage may be beneficial; Zhang et al. [23] reported $22 \%$ of patients (4 out of 18 patients) with periprosthetic infection still had positive cultures during the open biopsy procedure even after a formal irrigation and debridement (I\&D), implant resection, antibiotic spacer, and a course of antibiotics. Interestingly, three of the four patients had persistent $P$. acnes infection. They were treated with another I\&D, antibiotic spacer placement, and 6 weeks of culture specific antibiotics. With this protocol, the authors reported $100 \%$ eradication of infection in all 18 patients.

\section{Hematoma}

Hematoma formation occurrence is relatively common in RTSA, and the reported incidence ranges from 1 to $20 \%$ [2, $14,16,24]$. Although it does not affect the overall outcome, there is evidence to suggest it can increase the rate of infection. The design of the prosthesis inherently creates a larger dead space after RTSA compared to TSA. The glenosphere is placed more inferior which leads to increased acromiohumeral distance. Certain designs medialize the glenoid thereby placing the humerus in a more valgus position which can also create a larger dead space [2]. Furthermore, patients with severely deficient rotator cuffs potentially can have a larger dead space. Careful intraoperative hemostasis should be maintained and postoperative suction drain placement can be helpful to decrease the amount of hematoma formation. In the case of major hematoma formation, prompt surgical intervention should be considered.

\section{Dislocation}

Dislocation is one of the most common complications reported after RTSA (Fig. 1). Zumstein et al. reported the incidence of instability to be $4.7 \%(37 / 782)$ in a systematic review [13••], but the previously published incidence ranges from 2.4 to $31 \%$ [2, 14, 25-27]. A deltopectoral approach was used in $97.3 \%$ of the patients reported in Zumstein et al., and $87.5 \%$ needed reoperation with increased frequency in the revision group compared to primary arthroplasty. There are several contributing factors that can lead to dislocation or instability: lack of soft tissue tension, mechanical impingement, mismatch of the glenosphere and humeral socket, and improper version of the prosthesis. Adequate soft tissue tension is essential in the stability of the reverse shoulder. Vertical offset of the acromion-greater tuberosity distance and lateral offset of the tuberosity-glenoid distance should be recreated to achieve anatomic soft tissue tension [2]. The type of implant used can also influence soft tissue tensioning. In Grammontstyle prostheses, soft tissue tension can be achieved with a combination of inferior glenosphere placement, large glenosphere, and valgus neck-shaft angle. In a prosthesis with a laterally offset glenosphere, the increased lateral-medial soft

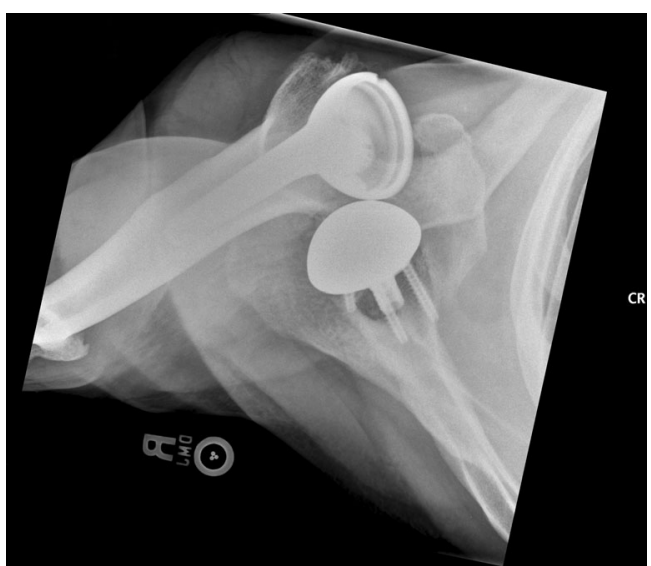

Fig. 1 Anterior dislocation after reverse shoulder arthroplasty. Patient was treated with closed reduction and eventual revision surgery 
tissue tension may decrease the need for tension in the vertical direction [2].

The role of subscapularis muscle in the setting of instability is currently controversial. Ackland et al. suggests that in RTSA patients, the superior aspect of muscle acts as an abductor while the inferior aspect acts like an adductor, leading to increased tension and stability $[12,28]$. This study has not been correlated in the clinical setting. Edwards et al. [29] evaluated 138 reverse shoulder arthroplasty patients with and without subscapularis repair. All postoperative dislocations were seen in the patients with irreparable subscapularis at the time of surgery. Risk factors for dislocation include proximal humerus nonunions, fixed glenohumeral dislocations, and failed prior arthroplasty or revisions. Chalmers et al. [30] also supported the above findings and reported increased risk of dislocation in males, BMI $>30 \mathrm{~kg} / \mathrm{m}^{2}$, subscapularis deficiency, and revision surgery after reverse shoulder.

In the setting of dislocation, immediate closed reduction should be followed by a period of sling immobilization and avoidance of extension, adduction, and internal rotation [2]. Patients should be placed in an abduction brace for 6 weeks. Malposition of implants and inadequate of soft tissue tensioning should be considered in cases of recurrent dislocation and revision surgery that include component exchange to a larger glenosphere and increasing the size of the humeral tray or thickness of the polyethylene insert may be necessary to increase the soft tissue tension to provide increased stability. In the subset of patients that have an increased risk for dislocation (male, higher BMI, irreparable subscapularis, and revision surgery), an abduction orthosis should be considered in the postoperative phase to decrease risk of dislocation [30].

\section{Scapular notching}

Scapular notching is the most commonly reported complication associated with RTSA. Notching is caused by direct mechanical impingement of the humeral prosthesis or metaphyseal bone on the inferior scapular neck. This leads to radiographic appearance of wear of the lateral pillar of the scapula immediately medial and progressively superior to the inferior aspect of the baseplate. Sirveaux et al. classified scapular notching based on the defect size seen on radiographs. Grade 1 defects are confined to the pillar, grade 2 extends to the lower screw, grade 3 compasses the screw, and grade 4 involves the central post under the baseplate [31]. Several factors are associated with developing notching: rotator cuff arthropathy and fatty infiltration of the infraspinatus, narrow acromiohumeral distance, and superiorly oriented glenoid [3]. Scapular notching was reported in $35 \%$ (277/782) of the patients in Zumstein et al. [13••] but the true incidence of notching varies and it is dependent upon the type of prosthesis used. Grammont-style prostheses, in which the glenosphere is placed on the face of the glenoid, has a reported incidence of notching that ranges from 51 to $96 \%$ [2, 8, 14, 15, 32-34]. New reports using a laterally offset glenosphere have reported notching incidence that ranges from 0 to $13 \%[1,2,35]$.

The clinical significance of scapular notching is controversial. Werner et al. reported on 58 consecutive patients who underwent RTSA with Delta III prosthesis to manage irreparable rotator cuff tears with average follow-up duration of 38 months. The study found scapular notching in $96 \%$ of the follow-up patients with even distribution of grades 1 and 2 versus grades 3 and 4 [14]. The authors did find any correlation between notching and objective or subjective clinical outcome. Wall et al. [15] reported 186 patients (191 shoulders) with a majority of them using the Delta III system. Fifty-seven percent of the patients (152 shoulders) who had radiographic follow-up had evidence of scapular notching. The mean constant score was 60.6 points in patients with notching compared to 58.7 in those without. The mean elevation was $132^{\circ}$ for patients with notching and $131^{\circ}$ for those without. There was no glenoid loosening in group of patients with notching. Cazeneuve et al. [26] reported 19 out of 36 patients who underwent RTSA with the Delta III prosthesis had scapular notching at a mean followup of 6.6 years. Twenty-three patients had radiographic evidence of loosening of the glenoid component and 1 had aseptic baseplate failure. Gutierrez et al.[36] used a virtual model of the RTSA to evaluate factors that may lead to inferior impingement. The authors concluded that the lateralized center of rotation was the most important variable in the avoidance of inferior impingement. Furthermore, Nyffeler et al. [37]. and Levigne et al. [34] suggested inferior placement of the glenosphere can also reduce the incidence of scapular notching. Li et al. [38] found that only inferior placement, inferior tilt of 15-30 , and lateralization best allowed impingement-free motion during external and internal rotation, which is associated with posterior and anterior notching, respectively.

Acromial/scapular spine fracture

Acromial fractures are infrequently seen in patients who underwent RTSA (Fig. 2). In the systematic review performed

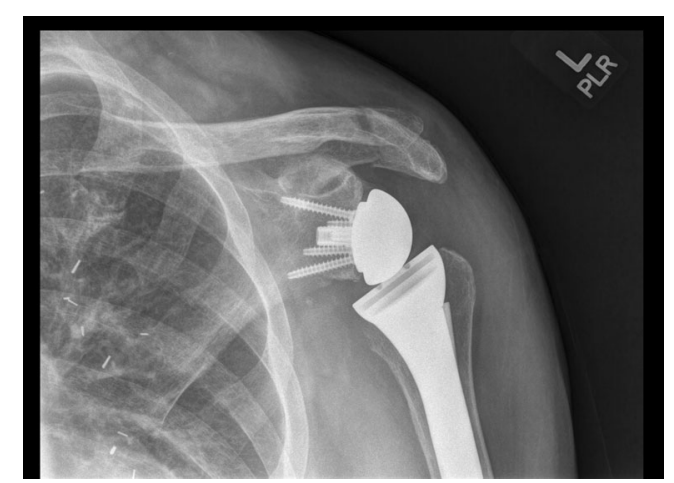

Fig. 2 Acromial fracture after reverse shoulder arthroplasty. Patient was treated with conservative management 
by Zumstein et al., the combined incidence of acromial and scapular spine fracture was $1.5 \%(12 / 782)$ [13••]. Patients typically report a sudden onset of lateral shoulder pain with associated decline in function. In patients who are candidates for RTSA, acromial erosion has already occurred due to the underlying humeral head. If the subscapularis is intact, the erosion may be more posterior and potentially affect the scapular spine. Postoperatively, due to increased deltoid tension and medialization of the center of rotation, the load across the acromion significantly increases. Levy et al. [39] classified acromial fractures into three types based on CT scans: type 1 involves a portion between the anterior and medial deltoid origin, type II involves at least the entire medial deltoid origin with a portion of the posterior deltoid, and type III involves the entire middle and posterior deltoid origin. All acromial fractures were treated with conservative management and resulted in limitation in function. Hattrup et al. [40] reported improved functional outcome results with conservative management of acromial and scapular spine fractures with greater of 1 year of follow-up. The authors cautioned against open reduction and internal fixation due to the challenges of stable internal fixation around this particular location. Crosby et al. [41] recommended conservative management in the acromial avulsion fractures and internal fixation in patients with scapular spine fracture to optimize outcome. Most acromial fractures can be treated conservatively but scapular spine fractures can lead to poor outcome with regard to constant score, active elevation, and subjective satisfaction, and surgical intervention may be required [2]. Osteoporosis along with a decrease in the acromial to tuberosity distance is major risk factors for acromial fractures. Increasing the acromial tilt on the radiographs and also using advanced imaging studies will help in the diagnosis [42].

\section{Conclusion}

Since the initial introduction by Grammont in France, the indications for RTSA have expanded and the number of procedures performed annually is expected to rise in the next several years. Complications associated with reverse arthroplasty include hematoma, neurological injury, dislocation, infection, fractures, and scapular notching. These continue to pose a significant challenge to surgeons, an increasing economic burden to society, and can lead to worsening patient satisfactions and outcomes. Recognizing both the preoperative risk factors and essential intraopative technical steps while understanding the mechanics and component design is essential to minimize patient morbidity and improve outcome. Future mutli-center trials and national registries are needed to further investigate the best approach and course of treatment in patients that present with complications after the reverse shoulder arthroplasty.

\section{Compliance with Ethics Guidelines}

Conflict of Interest Hanbing Zhou, Justin Chung, and Paul Yi have nothing to disclose.

Xinning Li reports compensation from Tornier and Mitek Sports Medicine, outside the submitted work.

Mark Price reports personal fees from DePuy Mitek outside the submitted work.

Human and Animal Rights and Informed Consent No human or animal studies were performed by the authors. This article does not contain any studies with human or animal subjects performed by any of the authors.

\section{References}

Papers of particular interest, published recently, have been highlighted as:

- Of importance

•• Of major importance

1. Frankle M et al. The reverse shoulder prosthesis for glenohumeral arthritis associated with severe rotator cuff deficiency. A minimum 2-year follow-up study of 60 patients. J Bone Joint Surg Am. 2005;87(8):1697-705.

2. Cheung E et al. Complications in reverse total shoulder arthroplasty. J Am Acad Orthop Surg. 2011;19(7):439-49.

3. Farshad M, Gerber C. Reverse total shoulder arthroplasty-from the most to the least common complication. Int Orthop. 2010;34(8): 1075-82.

4. Scarlat MM. Complications with reverse total shoulder arthroplasty and recent evolutions. Int Orthop. 2013;37(5):843-51.

5. Levy $\mathrm{J}$ et al. The use of the reverse shoulder prosthesis for the treatment of failed hemiarthroplasty for proximal humeral fracture. J Bone Joint Surg Am. 2007;89(2):292-300.

6. Guery $\mathrm{J}$ et al. Reverse total shoulder arthroplasty. Survivorship analysis of 80 replacements followed for 5 to 10 years. J Bone Joint Surg Am. 2006;88(8):1742-7.

7. Van Hoof T et al. 3D computerized model for measuring strain and displacement of the brachial plexus following placement of reverse shoulder prosthesis. Anat Rec (Hoboken). 2008;291(9):1173-85.

8. Boileau P et al. Grammont reverse prosthesis: design, rationale, and biomechanics. J Shoulder Elbow Surg. 2005;14(1 Suppl S):147S$61 \mathrm{~S}$.

9. Muh SJ et al. Early follow-up of reverse total shoulder arthroplasty in patients sixty years of age or younger. J Bone Joint Surg Am. 2013;95(20):1877-83. Specific article that investigates the early outcomes of reverse shoulder arthroplasty in a younger patient population.

10. Wingert NC, Beck JD, Harter GD. Avulsive axillary artery injury in reverse total shoulder arthroplasty. Orthopedics. 2014;37(1):e92-7.

11. Ladermann A et al. Injury to the axillary nerve after reverse shoulder arthroplasty: an anatomical study. Orthop Traumatol Surg Res. 2014;100(1):105-8.

12. Jarrett CD, Brown BT, Schmidt CC. Reverse shoulder arthroplasty. Orthop Clin North Am. 2013;44(3):389-408. $x$.

13.• Zumstein MA et al. Problems, complications, reoperations, and revisions in reverse total shoulder arthroplasty: a systematic review. 
J Shoulder Elbow Surg. 2011;20(1):146-57. A systematic review of the complications and revisions in reverse total shoulder arthroplasty.

14. Werner CM et al. Treatment of painful pseudoparesis due to irreparable rotator cuff dysfunction with the Delta III reverse-ball-andsocket total shoulder prosthesis. J Bone Joint Surg Am. 2005;87(7): 1476-86.

15. Wall B et al. Reverse total shoulder arthroplasty: a review of results according to etiology. J Bone Joint Surg Am. 2007;89(7):1476-85.

16. Cuff D et al. Reverse shoulder arthroplasty for the treatment of rotator cuff deficiency. J Bone Joint Surg Am. 2008;90(6):1244-51.

17. Cuff DJ et al. The treatment of deep shoulder infection and glenohumeral instability with debridement, reverse shoulder arthroplasty and postoperative antibiotics. J Bone Joint Surg (Br). 2008;90(3):336-42.

18. Cheung EV, Sperling JW, Cofield RH. Infection associated with hematoma formation after shoulder arthroplasty. Clin Orthop Relat Res. 2008;466(6):1363-7.

19. Zimmerli W, Trampuz A, Ochsner PE. Prosthetic-joint infections. N Engl J Med. 2004;351(16):1645-54.

20. Zavala JA et al. Management of deep infection after reverse total shoulder arthroplasty: a case series. J Shoulder Elbow Surg. 2012;21(10):1310-5.

21. Engesaeter LB et al. Antibiotic prophylaxis in total hip arthroplasty: effects of antibiotic prophylaxis systemically and in bone cement on the revision rate of 22,170 primary hip replacements followed 0 14 years in the Norwegian Arthroplasty Register. Acta Orthop Scand. 2003;74(6):644-51.

22. Beekman PD et al. One-stage revision for patients with a chronically infected reverse total shoulder replacement. J Bone Joint Surg (Br). 2010;92(6):817-22.

23. Zhang, A.L., et al., Management of deep postoperative shoulder infections: is there a role for open biopsy during staged treatment? J Shoulder Elbow Surg, 2014

24. Boileau P et al. Neer award 2005: the Grammont reverse shoulder prosthesis: results in cuff tear arthritis, fracture sequelae, and revision arthroplasty. J Shoulder Elbow Surg. 2006;15(5):527-40.

25. Bufquin $\mathrm{T}$ et al. Reverse shoulder arthroplasty for the treatment of three- and four-part fractures of the proximal humerus in the elderly: a prospective review of 43 cases with a short-term follow-up. J Bone Joint Surg (Br). 2007;89(4):516-20.

26. Cazeneuve JF, Cristofari DJ. The reverse shoulder prosthesis in the treatment of fractures of the proximal humerus in the elderly. J Bone Joint Surg (Br). 2010;92(4):535-9.

27. Gerber C, Pennington SD, Nyffeler RW. Reverse total shoulder arthroplasty. J Am Acad Orthop Surg. 2009;17(5):284-95.
28. Ackland DC et al. Moment arms of the shoulder musculature after reverse total shoulder arthroplasty. J Bone Joint Surg Am. 2010;92(5):1221-30.

29. Edwards TB et al. Subscapularis insufficiency and the risk of shoulder dislocation after reverse shoulder arthroplasty. J Shoulder Elbow Surg. 2009;18(6):892-6.

30. Chalmers PN et al. Early dislocation after reverse total shoulder arthroplasty. J Shoulder Elbow Surg. 2014;23(5):737-44.

31. Sirveaux F et al. Grammont inverted total shoulder arthroplasty in the treatment of glenohumeral osteoarthritis with massive rupture of the cuff. Results of a multicentre study of 80 shoulders. J Bone Joint Surg (Br). 2004;86(3):388-95.

32. Boulahia A et al. Early results of a reverse design prosthesis in the treatment of arthritis of the shoulder in elderly patients with a large rotator cuff tear. Orthopedics. 2002;25(2):129-33.

33. Simovitch RW et al. Predictors of scapular notching in patients managed with the Delta III reverse total shoulder replacement. J Bone Joint Surg Am. 2007;89(3):588-600.

34. Levigne $C$ et al. Scapular notching in reverse shoulder arthroplasty. J Shoulder Elbow Surg. 2008;17(6):925-35.

35. Mulieri $P$ et al. Reverse shoulder arthroplasty for the treatment of irreparable rotator cuff tear without glenohumeral arthritis. J Bone Joint Surg Am. 2010;92(15):2544-56.

36. Gutierrez $\mathrm{S}$ et al. Evaluation of abduction range of motion and avoidance of inferior scapular impingement in a reverse shoulder model. J Shoulder Elbow Surg. 2008;17(4):608-15.

37. Nyffeler RW, Werner CM, Gerber C. Biomechanical relevance of glenoid component positioning in the reverse Delta III total shoulder prosthesis. J Shoulder Elbow Surg. 2005;14(5):524-8.

38. Li X et al. Effects of glenosphere positioning on impingement-free internal and external rotation after reverse total shoulder arthroplasty. J Shoulder Elbow Surg. 2013;22(6):807-13.

39. Levy JC, Anderson C, Samson A. Classification of postoperative acromial fractures following reverse shoulder arthroplasty. J Bone Joint Surg Am. 2013;95(15):e104.

40. Hattrup, S.J., The influence of postoperative acromial and scapular spine fractures on the results of reverse shoulder arthroplasty. Orthopedics, 2010. 33(5).

41. Crosby LA, Hamilton A, Twiss T. Scapula fractures after reverse total shoulder arthroplasty: classification and treatment. Clin Orthop Relat Res. 2011;469(9):2544-9.

42. Otto RJ et al. Scapular fractures after reverse shoulder arthroplasty: evaluation of risk factors and the reliability of a proposed classification. J Shoulder Elbow Surg. 2013;22(11):1514-21. 